\title{
Centrifugal Compressor Surge and Speed Control
}

\author{
Jan Tommy Gravdahl, Member, IEEE, and Olav Egeland, Member, IEEE
}

\begin{abstract}
Previous work on stabilization of compressor surge is extended to include control of the angular velocity of the compressor. First a low-order centrifugal compressor model is presented where the states are mass flow, pressure rise, and rotational speed of the spool. Energy transfer considerations are used to develop a compressor characteristic. In order to stabilize equilibria to the left of the surge line, a close coupled valve is used in series with the compressor. Controllers for the valve pressure drop and spool speed are derived. Semiglobal exponential stability is proved using a Lyapunov argument.
\end{abstract}

Index Terms - Compressors, Lyapunov methods, modeling, surge control.

\section{INTRODUCTION}

C OMPRESSOR surge is an axisymmetric oscillation of the mass flow and pressure rise. Modeling and control of these oscillations is of considerable interest since surge limits the useful range of mass flows where the compressor operates stably. Large amplitude surge can also damage the compressor. Low-order models for surge in compression systems have been proposed by many authors, and a classical reference is [7]. However, the compression system model of [17] has been widely used for surge control design. It was derived for axial compression systems, but [19] showed that it is also applicable to centrifugal compressors. The model has two states, normalized mass flow and normalized pressure, and the compressor is treated as an actuator disc, with a third-order polynomial flow/pressure rise characteristic.

Over the last decade many papers covering the area of surge control have been published. A review can be found in [16]. Of many possible actuation schemes, closed coupled valve (CCV) control is considered one of the most promising [6], [18], [21], [25], [27]. Experimental results of CCV control is reported in [6] and [21]. Surge in a compression system can be explained by the throttle line crossing the compressor characteristic in an area of positive compressor characteristic slope. A close coupled valve is placed immediately downstream of the compressor (hence close coupled), and active control of the valve pressure drop is utilized to make the slope of the equivalent compressor (compressor in series with the valve) negative and thereby stabilizing the system.

This approach was used in [21], [25], and [27] for surge control of the model of [17]. Linear stability analysis was used in designing control laws resulting in local stability results. In [26] pressure disturbances were included in the analysis, and

Manuscript received November 25, 1997; revised July 28, 1998. Recommended by Associate Editor, M. Jankovic.

The authors are with the Department of Engineering Cybernetics, Norwegian University of Science and Technology, N-7034 Trondheim, Norway.

Publisher Item Identifier S 1063-6536(99)06452-0. a nonlinear CCV control law was designed for the model of [17] using the method of Lyapunov. By applying backstepping, [13] developed nonlinear surge controllers for the same model, but included disturbances both in mass flow and pressure. Global stability results were presented. In [14] certain passivity properties of the model were utilized in designing a CCV control law.

One drawback of CCV control is that the valve introduces a pressure drop in the compression system as discussed in [26]. When using the valve as a steady-state device, such as in [6], this loss may become unacceptably large. However, as pointed out in [25] and [26], a time varying valve will introduce considerably less pressure drop than a valve with constant pressure drop.

Since compressors are variable speed machines, it is of interest to investigate the influence of speed transients on the surge dynamics. Models describing this interaction were developed in [8] and [12] for axial compressors, and in [10] and [15] (a preliminary version of this paper) for centrifugal compressors. As surge can occur during acceleration of the compressor speed, it is of major concern to develop controllers that simultaneously can control both surge and compressor speed.

In this paper, a surge control law for variable speed centrifugal compressors is presented and analyzed. The speed is controlled with a PI-control law. Inspired by [9] and [29], we make a departure from the third order polynomial approximation of the compressor characteristic commonly used in the surge control literature. Fluid friction and incidence losses, as well as other losses, in the compressor stage are modeled, and a variable speed compressor characteristic is developed based on this. Both annular and vaned diffusers are studied.

Semiglobal exponential stability results for the proposed controllers are given using Lyapunovs method, and the results are confirmed through simulations.

\section{MODEL}

The centrifugal compressor consists essentially of a stationary inlet casing, a rotating impeller which imparts a high velocity to the gas, and a number of fixed diverging passages in which the gas is decelerated with a consequent rise in static pressure. The latter process is one of diffusion, and consequently, the part of the compressor containing the diverging passages is known as the diffuser, [2]. Fig. 1 is a diagrammatic sketch of the impeller and diffuser of a centrifugal compressor. The function of the inlet casing is to deliver gas to the impeller eye. A volute (also known as a scroll or a collector) may be fitted at the diffuser exit. Its 


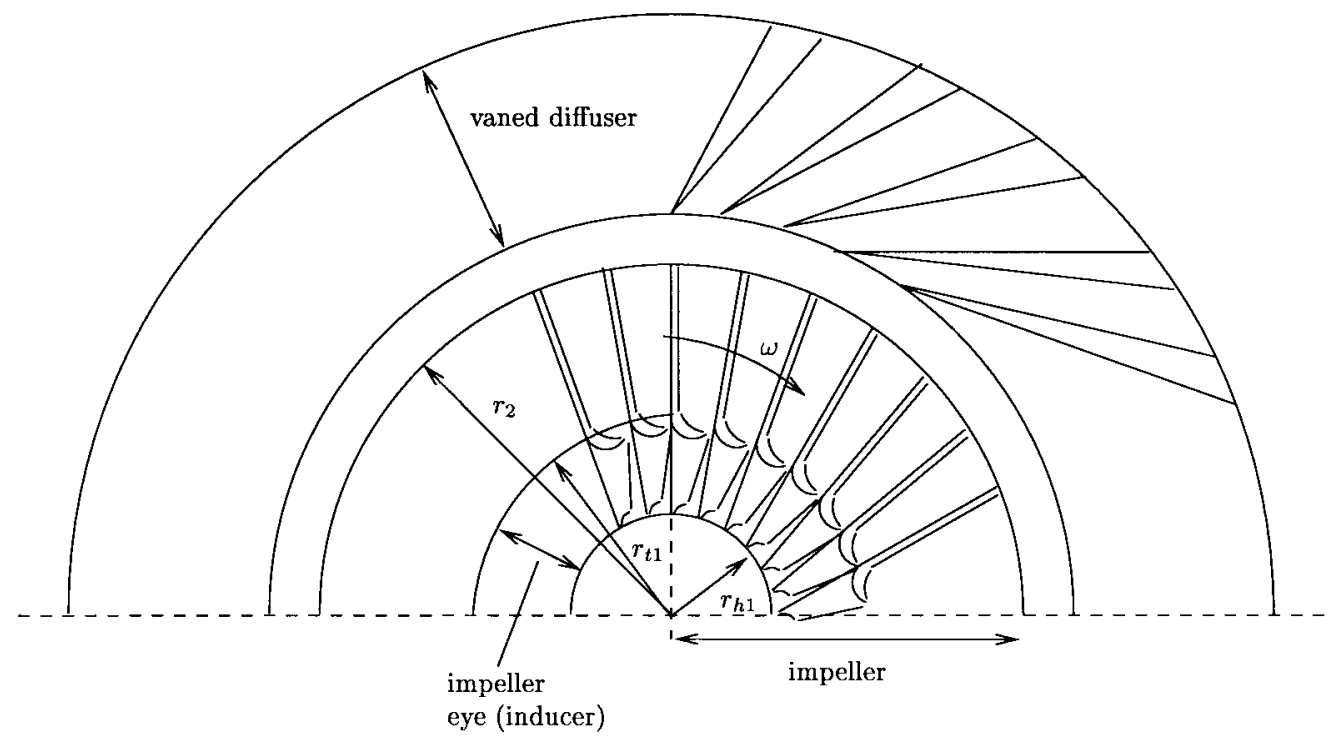

Fig. 1. Diagrammatic sketch of a radially vaned centrifugal compressor. Shown here with a vaned diffuser.

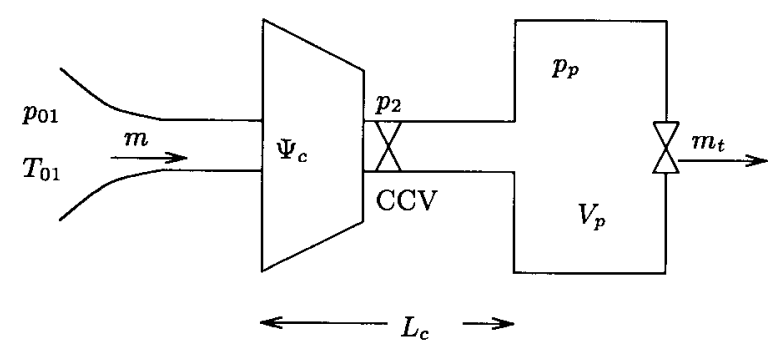

Fig. 2. Compression system.

function is simply to collect the diffuser exit flow, and to guide it as efficiently as possible to the compressor outlet, without impeding the effectiveness of the diffuser [29].

We are considering a compression system consisting of a centrifugal compressor, close coupled valve, compressor duct, plenum volume, and a throttle. The throttle can be regarded as a simplified model of a turbine. The system is shown in Fig. 2. The model to be used for controller design is in the form

$$
\begin{aligned}
\dot{p}_{p} & =\frac{a_{01}^{2}}{V_{p}}\left(m-m_{t}\right) \\
\dot{m} & =\frac{A_{1}}{L_{c}}\left(p_{2}-p_{p}\right) \\
\dot{\omega} & =\frac{1}{J}\left(\tau_{t}-\tau_{c}\right)
\end{aligned}
$$

where $m$ is the compressor mass flow, $p_{2}$ is the pressure downstream of the compressor, $a_{01}$ is the inlet stagnation sonic velocity, $L_{c}$ is the length of compressor and duct, $A_{1}$ is a reference area, $J$ is the spool moment of inertia, $\tau_{t}$ is the drive torque and $\tau_{c}$ is the compressor torque. The two first equations of (1) are equivalent to the model of [17], whereas the whole model (1) is similar to the model of [10]. It should also be noted that models similar to (1) are used in modeling the compressor in IC engine turbochargers, see [11]. In which case, $p_{p}$ would be the intake manifold or intercooler pressure.

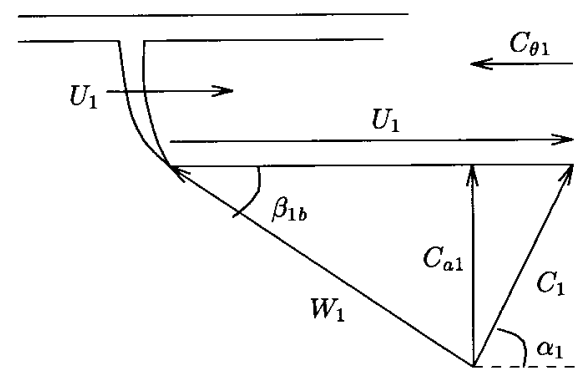

Fig. 3. Velocity triangle at inducer.

The angular speed $\omega$ of the compressor is included as a state in addition to mass flow and pressure rise which are the states in Greitzers surge model. The equation for $\dot{p}_{p}$ follows from the mass balance in the plenum, assuming the plenum process isentropic, and the equation for $\dot{m}$ follows from the impulse balance in the duct. In the following, the model (1) will be developed in detail. In particular, expressions must be found for the terms $p_{2}$ and $\tau_{c}$. It will also be shown that an expression for the compressor characteristic results from this derivation.

Incoming gas enters the impeller eye (the inducer) of the compressor with velocity $C_{1}$ (see Fig. 3 ). The mass flow $m$ and $C_{1}$ is given by

$$
C_{1}=\frac{1}{\rho_{01} A_{1}} m
$$

where $\rho_{01}$ is the constant stagnation inlet density. The tangential velocity $U_{1}$ of the inducer is calculated as

$$
U_{1}=\frac{D_{1}}{2} \omega=D_{1} \pi N
$$

where $\omega$ is the angular velocity of the impeller and $N$ is the number of revolutions per second. The average diameter $D_{1}$ is defined according to

$$
D_{1}^{2} \triangleq \frac{1}{2}\left(D_{t 1}^{2}+D_{h_{1}}^{2}\right)
$$




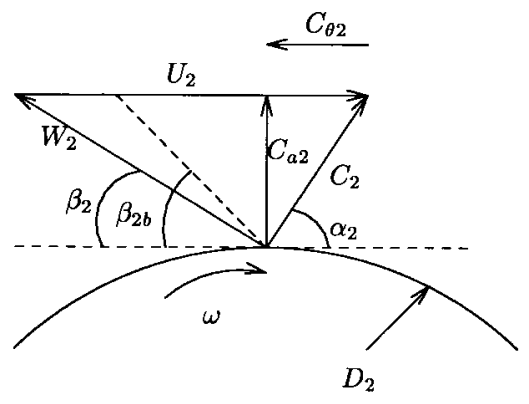

Fig. 4. Velocity triangle at impeller tip.

where $D_{t 1}$ and $D_{h_{1}}$ are the diameters at inducer tip and hub casing, respectively. The circle with diameter $D_{1}$ and area $A_{1}$ divides the inducer in two annuli of equal area. The gas leaves the impeller at the impeller tip with velocity $C_{2}$ as shown in Fig. 4. The diameter at the impeller tip is $D_{2}$ and the tangential tip velocity is $U_{2}$.

\section{ENERGY TRANSFER}

\section{A. Ideal Energy Transfer}

For turbomachines, applied torque equals the change in angular momentum of the fluid

$$
\tau_{c}=m\left(r_{2} C_{\theta 2}-r_{1} C_{\theta 1}\right)
$$

where $\tau_{c}$ is the compressor torque, $r_{1}=\left(D_{1} / 2\right), r_{2}=\left(D_{2} / 2\right)$ and $C_{\theta 2}$ is the tangential component of the gas velocity $C_{2}$. Power delivered to the fluid is

$$
\begin{aligned}
\dot{W}_{c} & =\omega \tau_{c}=\omega m\left(r_{2} C_{\theta 2}-r_{1} C_{\theta 1}\right) \\
& =m\left(U_{2} C_{\theta 2}-U_{1} C_{\theta 1}\right)=m \Delta h_{0 c, \text { ideal }}
\end{aligned}
$$

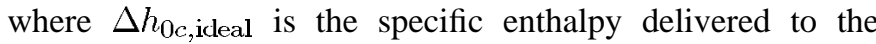
fluid without taking account for losses. Equation (6) is known as Euler's pump equation. For simplicity, the following two assumptions are made. A radially vaned (no backsweep) impeller is considered with $\beta_{2 b}=90^{\circ}$, and there is no prewhirl, that is $\alpha_{1}=90^{\circ} \Rightarrow C_{\theta 1}=0$. It is to be emphasized that these assumptions are made only to simplify the analysis, the results to follow can easily be generalized by omitting these assumptions.

For an ideal radially vaned impeller, the whirl or tangential component $C_{\theta 2}$ of the gas velocity leaving the impeller tip should equal $U_{2}$. However, due to the inertia of the gas between the impeller blades, the tangential gas velocity tends to be less than $U_{2}$. This effect is known as slip. The flow is deflected away from the direction of rotation of the impeller, which it leaves at an angle smaller than the vane angle. The slip factor is defined as

$$
\sigma \triangleq \frac{C_{\theta 2}}{U_{2}}
$$

which is a positive number less than unity. The slip factor depends largely on the number of impeller blades, but also on the passage geometry, the impeller eye tip to exit diameter ratio and mass flow rate. There exists many approximations for the slip factor, the one appropriate to radial vaned impellers which seems to agree best with experiment [2] is that due to Stanitz: $\sigma \approx 1-2 / n_{b}$, where $n_{b}$ is the number of compressor blades.

We are now able to compute the compressor torque $\tau_{c}$ needed in the model (1). Using the assumption of no prewhirl and (5), the compressor torque is

$$
\tau_{c}^{+}=m r_{2} C_{\theta 2}=m r_{2} \sigma U_{2}, \quad m>0 .
$$

The torque calculated in (8) is for forward flow. However, the compressor may enter deep surge, that is reversal of flow, and there is need for an expression for the compressor torque at negative mass flow. It will be assumed that the compressor torque of a centrifugal compressor in reversed flow can be calculated by the use of Euler's turbine equation

$$
\tau_{c}^{-}=m\left(r_{1} C_{\theta 1}-r_{2} C_{\theta 2}\right)=-m r_{2} \sigma U_{2}, \quad m<0 .
$$

Combination of (8) and (9) gives

$$
\tau_{c}=|m| r_{2} \sigma U_{2}
$$

which is in accordance with the compressor torque used in [10].

A resistive torque is also set up by the rotation of the impeller in the gas. This can be modeled as the rotation of a disc in a fluid, and is treated in detail in [9].

From (6), and using (7), we have that the ideal specific enthalpy delivered to the fluid is

$$
\Delta h_{0 c, \text { ideal }}=\frac{\dot{W}_{c, \text { ideal }}}{m}=\sigma U_{2}^{2} .
$$

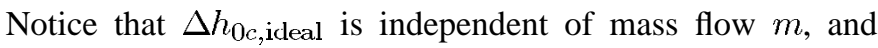
ideally we would have the same energy transfer for all mass flows (if backswept impeller blades, $\beta_{2 b}<90^{\circ}$, were

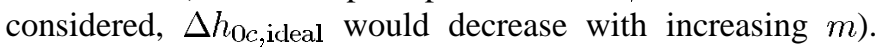
However, due to various losses, the energy transfer is not constant, and we now include this in the analysis. According to [29], [9], [23] and other authors, the two major losses, expressed as specific enthalpies, are the following.

1) Incidence losses in impeller and diffuser, $\Delta h_{i i}$ and $\Delta h_{i d}$.

2) Friction losses in impeller and diffuser, $\Delta h_{f i}$ and $\Delta h_{f d}$.

The incidence losses and fluid friction losses play an important role in determining the region of stable operation for the compressor. Other losses, such as back flow losses, clearance losses, and losses in the volute will be taken into account when computing the efficiency of the compressor. There also exist other losses such as inlet casing losses, mixing losses and leakage losses, but these will be ignored in the following. For a further treatment on this topic, some references are [1], [20], [32], and [3].

\section{B. Incidence Losses}

The losses due to incidence onto the rotor and vaned diffuser play an important role in shaping the compressor characteristic. There exists several methods of modeling this loss, and a 
comparative study is given in [31]. The two most widely used approaches are the following.

1) The so called "NASA shock loss theory" reported in [29] and [31], which is based upon the tangential component of kinetic energy being destroyed.

2) A constant pressure incidence model reported by [31] where it is assumed that the flow just inside the blades has adapted to the blades via a constant pressure process.

For centrifugal compressors, the differences between the models are small [29]. According to [31], the main difference lies in the prediction of the incidence angle at which zero loss occurs. For model (1) zero loss is predicted when the flow angle at the inlet equals the blade angle. This is not the case for model (2). Based on this, and the simplicity of (1), the NASA shock loss theory is used here.

Depending on the mass flow being lower or higher than the design flow, positive or negative stall is said to occur. The use of model (1) leads to a loss varying with the square of the mass flow, symmetrical about the design flow. In [9] it is stated that the incidence loss in practice increase more rapidly with reduction of flow below design flow, than with increase of flow above the design flow. This would lead to a steeper compressor characteristic below the design point than above, but for simplicity this will not be treated further here. According to [28], such a characteristic is said to be right skew.

1) Impeller: The velocity of the incoming gas relative to the inducer is denoted $W_{1}$. In off-design operation there will be a mismatch between the fixed blade angle $\beta_{1 b}$ and the direction of the gas stream $\beta_{1}=\beta_{1}\left(U_{1}, C_{1}\right)$, as shown in Fig. 5. The angle of incidence is defined by

$$
\beta_{i} \triangleq \beta_{1 b}-\beta_{1} .
$$

As the gas hits the inducer, its velocity instantaneously changes its direction to comply with the blade inlet angle $\beta_{1 b}$. The direction is changed from $\beta_{1}$ to $\beta_{1 b}$, and the kinetic energy associated with the tangential component $W_{\theta 1}$ of the velocity is lost. That is, the incidence loss can be expressed as

$$
\Delta h_{i i}=\frac{W_{\theta 1}^{2}}{2} .
$$

The incidence loss model in (13) is a simple one-dimensional model. According to [9], it approximates the loss owing to boundary-layer build-up for low angles of incidence and the increased losses resulting from flow separation at higher angles of incidence. As [9] points out, the term shock loss is misleading as nothing akin to shock occurs in practice, but the simple notion of shock is used to explain the shape of the compressor characteristic. From Fig. 5 it is easily seen that

$$
\cos \beta_{1}=\frac{U_{1}-C_{\theta 1}}{W_{1}} \text { and } \sin \beta_{1}=\frac{C_{a 1}}{W_{1}} .
$$

Furthermore,

$$
\begin{aligned}
W_{\theta 1} & =\frac{\sin \left(\beta_{1 b}-\beta_{1}\right)}{\sin \beta_{1 b}} W_{1} \\
& =\left(\cos \beta_{1}-\cot \beta_{1 b} \sin \beta_{1}\right) W_{1} .
\end{aligned}
$$

Combining (14) and (15) gives

$$
W_{\theta 1}=U_{1}-C_{\theta 1}-\cot \beta_{1 b} C_{a 1} .
$$

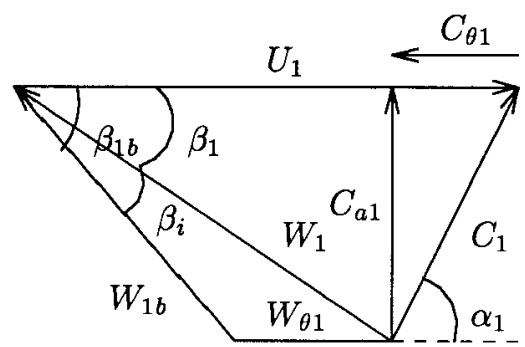

Fig. 5. Incidence angles at inducer.

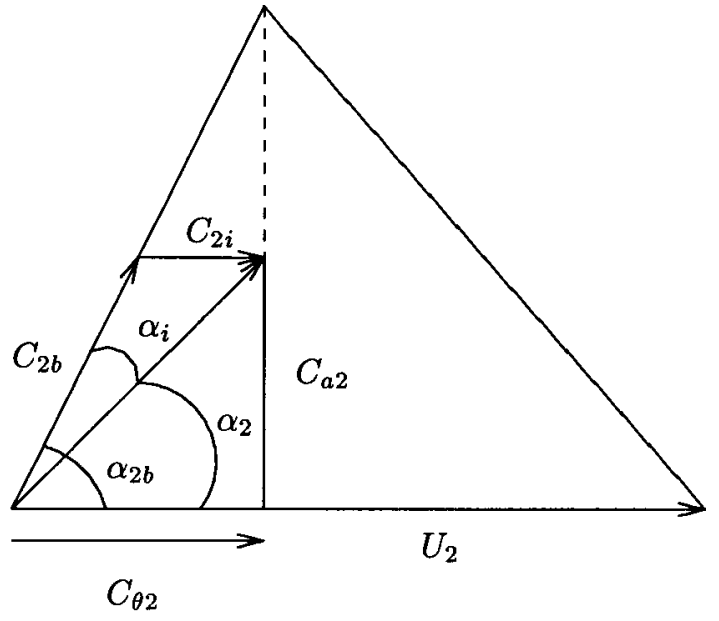

Fig. 6. Incidence angles at diffuser.

and the incidence loss (13) can be written

$$
\begin{aligned}
\Delta h_{i} & =\frac{1}{2}\left(U_{1}-C_{\theta 1}-\cot \beta_{1 b} C_{a 1}\right)^{2} \\
& =\frac{1}{2}\left(U_{1}-\frac{\cot \beta_{1 b} m}{\rho_{01} A_{1}}\right)^{2}
\end{aligned}
$$

where the second equality if found using (2). Similar results are presented in $[9$, ch. 5].

2) Diffuser: According to [29], the losses in the vaned diffuser can be modeled with friction/incidence losses in a similar manner as in the impeller. Similar to the inducer incidence loss, it is assumed that the velocity of the fluid entering the diffuser is instantaneously changed to comply with the fixed diffuser inlet angle $\alpha_{2 b}$. The direction is changed from $\alpha_{2}$ to $\alpha_{2 b}$, and the kinetic energy associated with the tangential component $C_{2 i}$ of the velocity is lost, see Fig. 6. That is, the incidence loss can be expressed as

$$
\Delta h_{i d}=\frac{C_{2 i}^{2}}{2} .
$$

Using Fig. 6 and (7) it is seen that

$$
\Delta h_{i d}=\frac{1}{2}\left(\sigma U_{2}-\cot \alpha_{2 b} C_{a 2}\right)^{2} .
$$

For simplicity the choice ${ }^{1} C_{a 1}=C_{a 2}$ is made. The diffuser inlet angle $\alpha_{2 b}$, is now designed such that there is minimum

\footnotetext{
${ }^{1}$ This is a design choice, and other choices will lead to different expression for the diffuser angle $\alpha_{2 b}$.
} 
incidence loss in both impeller and diffuser for the same mass flow $m$. For $\beta_{i}=0$, we have that

$$
U_{1}=C_{a 1} \cot \beta_{1 b} \Rightarrow C_{a 2}=U_{1} \tan \beta_{1 b} .
$$

From Fig. 6 and (20) it follows that

$$
\tan \alpha_{2 b}=\frac{C_{a 2}}{C_{\theta 2}}=\frac{U_{1} \tan \beta_{1 b}}{\sigma U_{2}}
$$

and

$$
\alpha_{2 b}=\operatorname{atan}\left(\frac{D_{1} \tan \beta_{1 b}}{\sigma D_{2}}\right)
$$

and consequently the diffuser incidence loss (19) can be written

$$
\Delta h_{i d}=\frac{1}{2}\left(\frac{\sigma D_{2} U_{1}}{D_{1}}-\frac{m \cot \alpha_{2 b}}{\rho_{01} A_{1}}\right)
$$

\section{Frictional Losses}

According to [9], loss due to friction in the impeller can be calculated as

$$
\Delta h_{f}=C_{h} \frac{l}{D} \frac{W_{1 b}^{2}}{2}
$$

where $C_{h}$ is the surface friction loss coefficient, ${ }^{2} l$ is the mean channel length and $D$ is the mean hydraulic channel diameter. This friction loss is actually calculated for constant area pipes of circular cross section. The friction loss coefficient $C_{h}$ is defined as [29]

$$
C_{h}=4 f
$$

where the friction factor $f$ depends on the Reynolds number. Many different formulas for the friction factor have been published (see, e.g., [9] or [30]). Here we will use Blasius' formula

$$
f=0.3164(\mathrm{Re})^{-0.25}
$$

which was found empirically for turbulent flow in smooth pipes with Reynolds number Re below 100 [30]. The mean hydraulic channel diameter $D$ is defined as

$$
D=\frac{4 A}{a}
$$

where the cross section area $A$ and perimeter $a$ are mean values for the passage. The mean hydraulic diameter $D$ corresponds to a circle with area $A$ and perimeter $a$. Although the passages between the blades in the compressor are neither circular nor of constant area, [1] reports of good agreement between theory and measurement using (26).

Using Fig. 5, it is seen that

$$
\frac{W_{1 b}}{\sin \beta_{1}}=\frac{W_{1}}{\sin \beta_{1 b}}
$$

and using $\sin \beta_{1}=\left(C_{a 1} / W_{1}\right)$, we get

$$
W_{1 b}=\frac{C_{1}}{\sin \beta_{1 b}} .
$$

\footnotetext{
${ }^{2}$ According to [29] diffusion losses in the impeller are small compared to impeller friction losses, but they may be included in the analysis by choosing $C_{h}$ to suit.
}

Inserting (2) and (29) in (24) gives

$$
\Delta h_{f}=\frac{C_{h} l}{2 D \rho_{1}^{2} A_{1}^{2} \sin ^{2} \beta_{1 b}} m^{2}=k_{f i} m^{2} .
$$

As can be seen from (30), the friction losses are quadratic in mass flow and independent of wheel speed $U$. Equation (30) represents the loss due to friction of a mass flow $m$ through a pipe of hydraulic diameter $D$.

The loss due to fluid friction in the diffuser can be modeled in a similar manner as in the impeller

$$
\Delta h_{f d}=k_{f d} m^{2} .
$$

In the vaned diffuser a pipe friction loss is calculated for each diffuser passage.

\section{Efficiency}

The isentropic efficiency of the compressor is defined as (see, e.g., [3])

$$
\eta_{i}\left(m, U_{1}\right)=\frac{\Delta h_{0 c, \text { ideal }}}{\Delta h_{0 c, \text { ideal }}+\Delta h_{\text {loss }}}
$$

where, in this paper

$$
\Delta h_{\mathrm{loss}}=\Delta h_{i f}+\Delta h_{i i}+\Delta h_{d f}+\Delta h_{d i} .
$$

Furthermore, the efficiency will be corrected with losses in the volute and the additional losses arising from clearance and backflow. The efficiency is also dependent on the ability of the diffuser to convert the kinetic energy of the flow into pressure. Collection the various losses, the isentropic efficiency from equation (34) is adjusted to

$$
\begin{aligned}
\eta_{i}\left(m, U_{1}\right)= & \frac{\Delta h_{0 c, \text { ideal }}}{\Delta h_{0 c, \text { ideal }}+\Delta h_{\text {loss }}} \\
& -\Delta \eta_{b f}-\Delta \eta_{c}-\Delta \eta_{v}-\Delta \eta_{d} .
\end{aligned}
$$

According to [24], the clearance loss $\Delta \eta_{c}$ of a centrifugal compressor can be approximated by $\Delta \eta_{c}=0.3\left(l_{c l} / b\right)$, where $l_{c l}$ is the axial clearance and $b$ is the impeller tip width. The backflow loss $\Delta \eta_{b f}$ occurs because the compressor has to reprocess the fluid that has been reinjected into the impeller due to pressure gradients existing in the impeller tip region. Due to the lack of accurate modeling of this loss, [29] suggest a loss of three points of efficiency as typical: $\Delta \eta_{b f}=0.03$. In the volute a loss will take place mainly due to the inability of the volute to use the radial kinetic energy out of the diffuser. In [3] this loss is assumed to lie within two to five point of efficiency: $0.02 \leq \Delta \eta_{v} \leq 0.05$. This loss is likely to be higher for compressor with a vaned diffuser than with an annular diffuser, as a larger part of the total kinetic energy at the outlet of the vaned diffuser is in the radial direction [3]. A more comprehensive treatment of loss in volutes can be found in [22]. The deceleration in the diffuser can be achieved more or less efficient depending on the construction of the diffuser. The efficiency drop $\Delta \eta_{d}$ due to inadequate diffusion is dependent on the pressure recovery coefficient, see, e.g., [3] or [29], but for simplicity $\Delta \eta_{d}$ will be considered constant here. According to [29], vaned diffusers offer a two to seven points increase in efficiency compared to annular diffusers. 


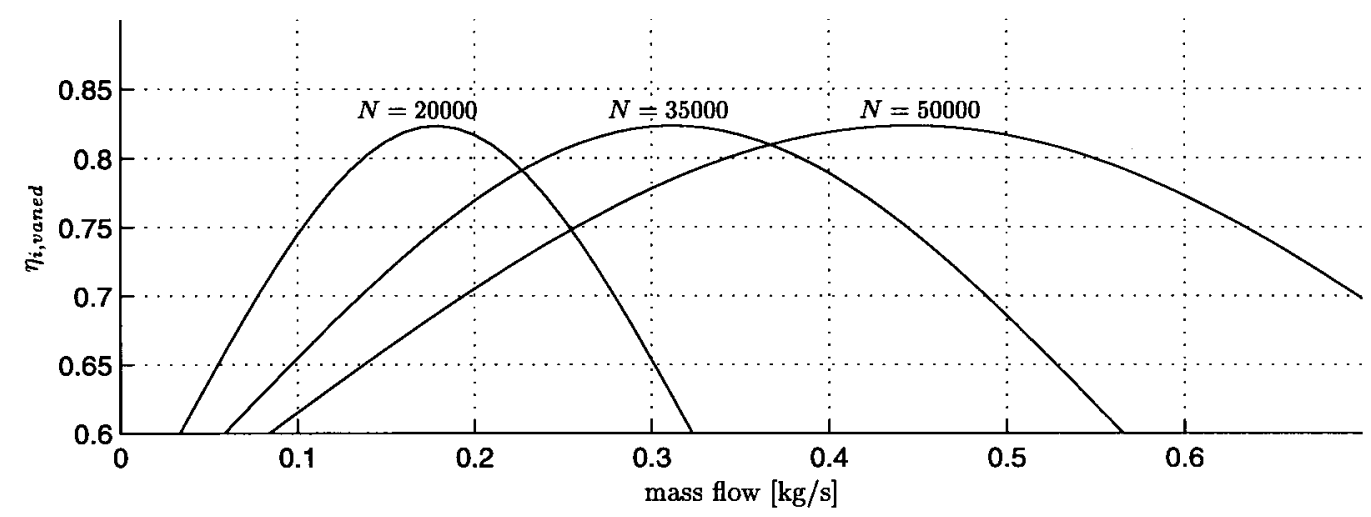

(a)

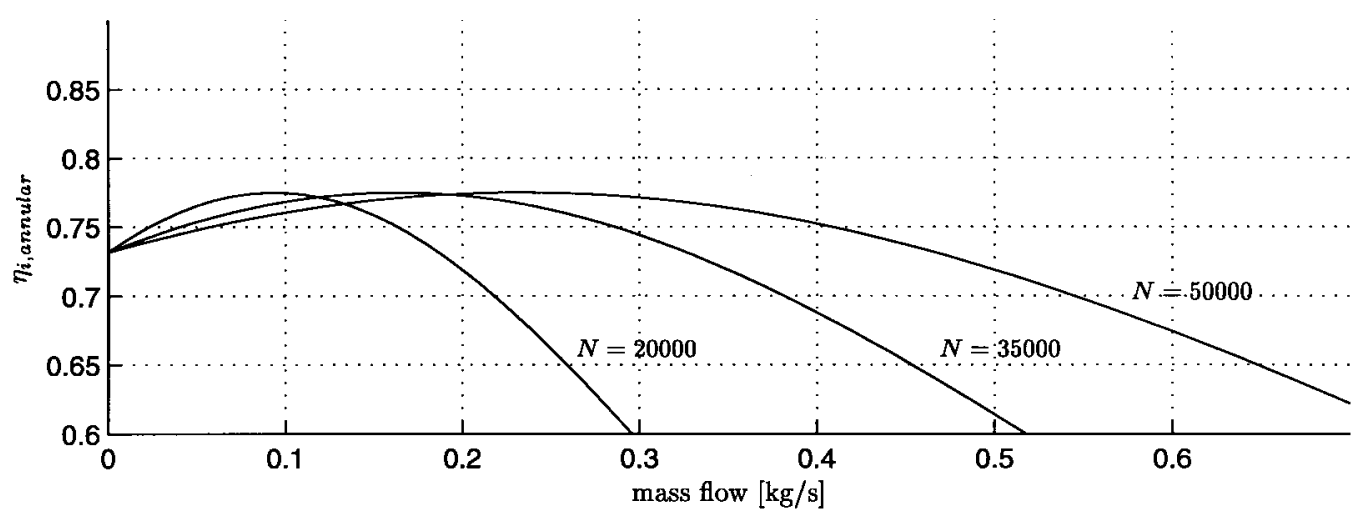

(b)

Fig. 7. Efficiencies for compressor with (a) vaned and (b) annular diffuser.

In Fig. 7, the efficiency $\eta_{i}$ is plotted. In the upper plot, the compressor is equipped with a vaned diffuser and in the lower plot with a annular diffuser. As can be seen, the vaned diffuser offer a higher efficiency, but a narrower range of flow compared with the annular diffuser.

\section{ENERgy Transfer AND PRessure Rise}

The total specific energy transfer can be calculated by subtracting (30), (17), (23), and (31) from (11)

$$
\Delta h_{0 c}\left(U_{1}, m\right)=\Delta h_{0 c, \text { ideal }}-\Delta h_{i f}-\Delta h_{i i}-\Delta h_{d f}-\Delta h_{d i} .
$$

$\Delta h_{0 c}$ is a second-degree polynomial in $m$, and as opposed to the ideal case, we see that energy transfer to the fluid is varying with mass flow $m$. This is shown in Fig. 8 .

To find an expression for the pressure rise we now need a relation between pressure rise and energy transfer. For forward flow, the pressure rise is modeled as

$$
\begin{aligned}
p_{2} & =\left(1+\frac{\eta_{i}\left(m, U_{1}\right) \Delta h_{0 c, \text { ideal }}}{T_{01} c_{p}}\right)^{\gamma / \gamma-1} \\
p_{01} & =\Psi_{c}\left(U_{1}, m\right) p_{01}
\end{aligned}
$$

where the losses have been taken into account, and $\Psi_{c}\left(U_{1}, m\right)$ is the compressor characteristic. In order to model the compressor pressure rise for negative mass flow, which will be encountered during deep surge, it is assumed that the pressure rise is proportional to the square of the mass flow for $m<0$, that is

$$
\begin{aligned}
& \Psi_{c}\left(U_{1}, m\right) \\
& \quad= \begin{cases}c_{n} m^{2}+\psi_{c 0}\left(U_{1}\right), & m \leq 0 \\
\left(1+\frac{\eta_{i}\left(m, U_{1}\right) \Delta h_{0 c, \text { ideal }}}{T_{01} c_{p}}\right)^{\gamma / \gamma-1}, & m>0\end{cases}
\end{aligned}
$$

where the choice

$$
\psi_{c 0}\left(U_{1}\right)=\left.\left(1+\frac{\eta_{i}\left(m, U_{1}\right) \Delta h_{0 c, \text { ideal }}}{T_{01} c_{p}}\right)^{\gamma / \gamma-1}\right|_{m=0}
$$

of the shut off value $\psi_{c 0}$ ensures that $\Psi_{c}\left(U_{1}, m\right)$ is continuous in $m$. According to [33] and [4], the back-flow characteristic defines the resistance which the rotating blades offer to flow in reversed direction. In [4] it is stated that in reversed flow the compressor can be regarded as a throttling device with a positive pressure bias. A compressor characteristic for both positive and negative flow is shown in Fig. 11.

A quadratic characteristic for reversed flow is also used in [19]. It is widely accepted in the literature (see, e.g., [18]) that the compressor characteristic has a negative slope for negative mass flow. This slope depends on the choice of the constant $c_{n}$.

We now have an expression for the pressure $p_{2}$ needed in the model (1). Notice that for each speed $N$, both the pressure rise (36) and the efficiency (34) reach maximum for the same value of mass flow $m$. Thus, the maximum efficiency is reached on the surge line, stressing the need for active control in order to be able to operate safely in the neighborhood of the surge line. 

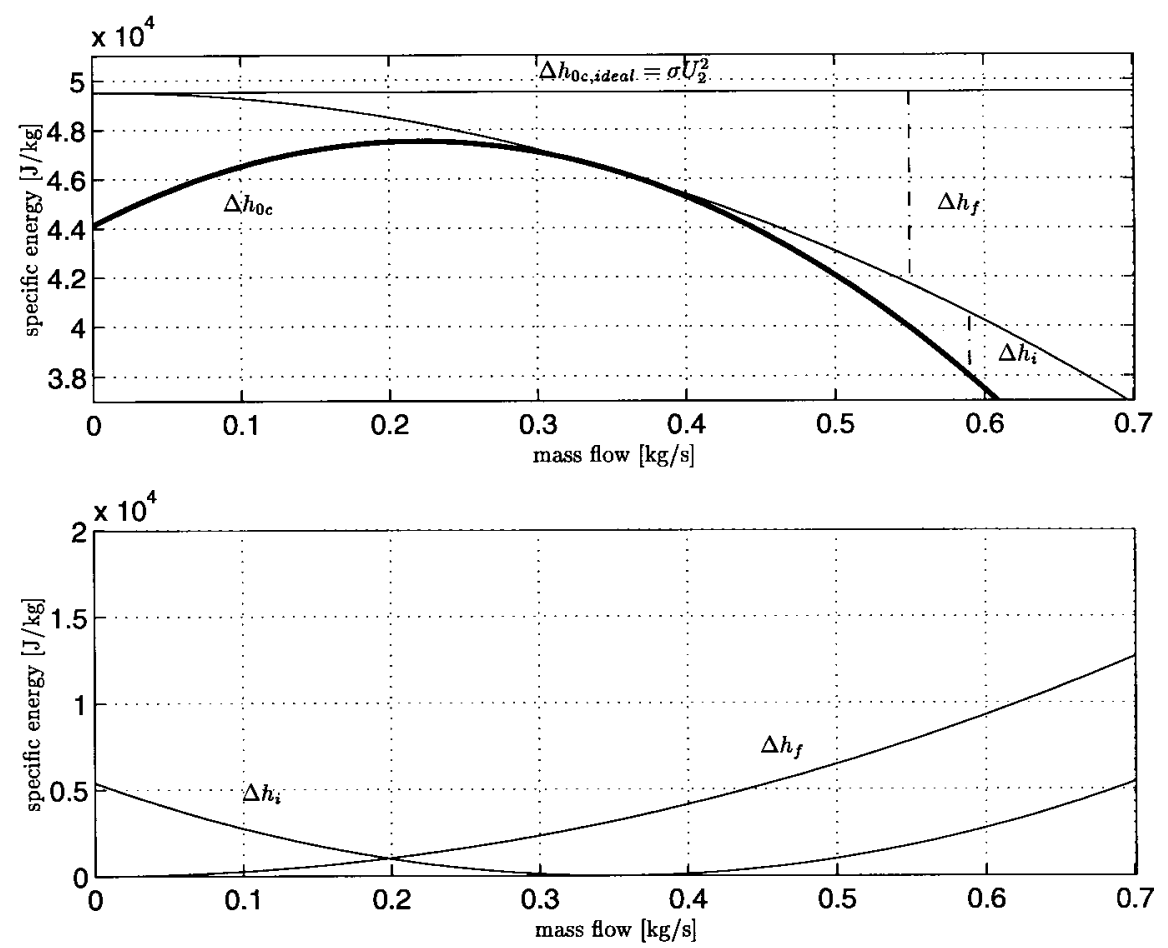

Fig. 8. Energy transfer for $N=35000 \mathrm{r} / \mathrm{min}$. Compressor with annular diffuser.
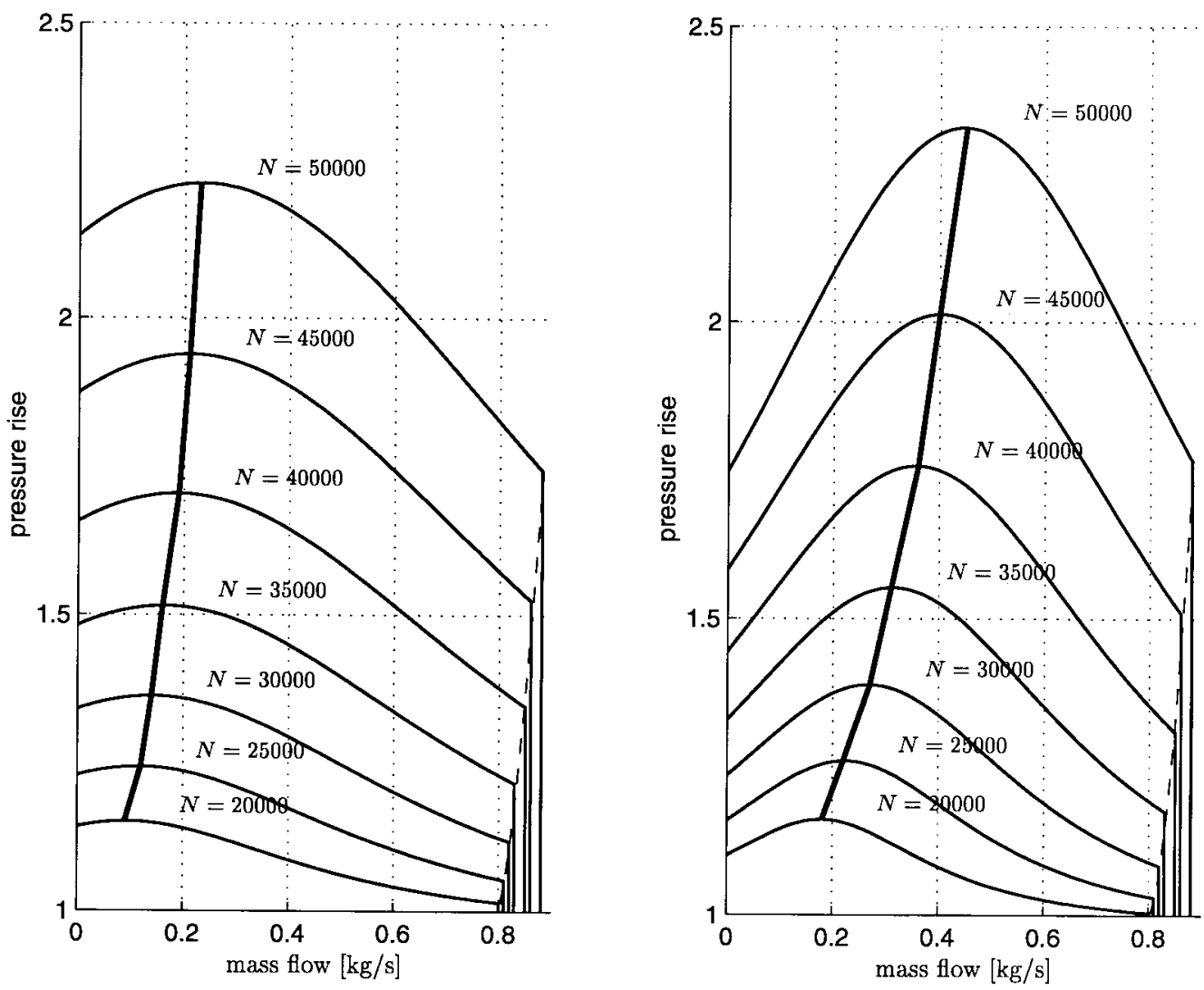

Fig. 9. Centrifugal compressor characteristic. The left plot is for a annular diffuser, and the right for a vaned diffuser.

The inlet stagnation temperature $T_{01}$, specific heat capacity $c_{p}$ and $\gamma$ are assumed constant. The ideal energy transfer and the losses are shown for a compressor with annular diffuser in Fig. 8. In the case of vaned diffuser, the curves look similar, but with a steeper slope du to the incidence losses at the diffuser. The curves are calculated for a compressor speed of $N=35000 \mathrm{r} / \mathrm{min}$. The compressor pressure characteristic as calculated from (36) is shown in Fig. 9. 
In Figs. 8 and 9 the numerical values for the compressor parameters are taken from [10]. The surge line is the line in the compressor map that divides the map into an area of stable compressor operation and unstable (surge) operation. The line passes through the local maxima of the constant speed lines in the map, and is drawn with a solid line in Fig. 9.

When the flow reaches sonic velocity at some cross section of the compression system, the flow chokes. Assuming isentropic flow, [5] calculated the choking flow for the components most likely to choke in centrifugal compressors, the impeller eye (the inducer) and at the entry of the diffuser. In this paper it is assumed that choking takes place in the impeller eye.

The effect of choking can be seen in Fig. 9, where a choke line, also known as a stone wall [23], has been drawn. In this paper, the effect of choking is treated in a approximate manner. Due to sonic effects, the pressure rise would fall off more gradually when approaching the stone wall than shown in Fig. 9. The choking flow is given as

$$
\begin{aligned}
& m_{\text {choke }}\left(U_{1}\right) \\
& =A_{1} \rho_{01} a_{01}\left[\frac{2+(\gamma-1)\left(\frac{U_{1}}{a_{01}}\right)^{2}}{\gamma+1}\right]^{(\gamma+1) / 2(\gamma-1)}
\end{aligned}
$$

where $\rho_{01}=p_{01} / R T_{01}$ is the inlet stagnation density and $a_{01}=\sqrt{\gamma R T_{01}}$ is the inlet stagnation sonic velocity. It is seen that the choking mass flow is dependent on blade speed $U_{1}$. Thus the impeller can accept a greater limiting mass flow rate at higher rotational speeds.

\section{DYNAMIC MODEL}

To complete the dynamic model (1), an expression for the throttle mass flow is needed. The mass flow $m_{t}$ through the throttle is modeled as

$$
m_{t}=k_{t} \sqrt{p_{p}-p_{01}}
$$

where $k_{t}$ is a parameter proportional to throttle opening and $p_{p}$ is the plenum pressure. The momentum balance of the spool is

$$
J \dot{\omega}=\tau_{t}-\tau_{c} .
$$

Using (3) it is seen that

$$
\omega=\frac{2 U_{1}}{D_{1}} \Rightarrow \dot{\omega}=\frac{2 \dot{U}_{1}}{D_{1}}
$$

and thus we get a differential equation for $U_{1}$

$$
\dot{U}_{1}=\frac{D_{1}}{2 J}\left(\tau_{t}-\tau_{c}\right) .
$$

The drive torque $\tau_{t}$ may be delivered by a turbine, and will be used as a control variable for speed control. The compressor and spool can only rotate in one direction, and the speed is assumed upper bounded

$$
0 \leq U_{1}(t)<U_{m}
$$

Assumption (44) is a technicality needed in the stability proof, and is not crucial to the derivation of the model. Using (10) and
(43), and inserting (36) and (40) in (1), we get the following dynamic model for the compression system ${ }^{3}$

$$
\begin{aligned}
\dot{p}_{p} & =\frac{a_{01}^{2}}{V_{p}}\left(m-k_{t} \sqrt{p_{p}-p_{01}}\right) \\
\dot{m} & =\frac{A}{L}\left(\left(1+\frac{\eta_{i}\left(m, U_{1}\right) \Delta h_{0 c, \text { ideal }}}{T_{01} c_{p}}\right)^{\gamma / \gamma-1} p_{01}-p_{p}\right) \\
\dot{U}_{1} & =\frac{D_{1}}{2 J}\left(\tau_{t}-\tau_{c}\right) .
\end{aligned}
$$

\section{Surge Control IDEA}

It is well known that the reason for equilibria to the left of the surge line being unstable, and causing the compressor to go into surge, is the positive slope of the characteristic in this area. From Fig. 8 it is seen that the positive slope is due to the incidence losses at low mass flows. From the expression for the incidence loss, (17), it is clear that a variable blade angle $\beta_{1 b}$ would make it possible to minimize the incidence losses over a range of mass flows. Thus variable inducer blades might be used as a means of surge stabilization.

On the other hand, the maximum energy transfer and minimum incidence loss do not occur for the same mass flow. This is due to the friction losses. The friction shifts the point of maximum energy transfer, and consequently pressure rise, to the left of the point of minimum incidence loss. From this we conclude that the friction losses in fact have a stabilizing effect, and introducing additional fluid friction would move the point of maximum energy transfer to the left. The effect of this is that the surge line will be shifted to the left, and the area of stable compressor operation is expanded.

This motivates us to introduce a valve in series with compressor. The pressure drop over this valve will serve as the control variable, and it will be used to introduce additional friction at low mass flows in order to avoid surge. The CCV will be regarded as a idealized actuator, a device which can produce a desired pressure drop.

\section{Controller Design And Stability Analysis}

The equivalent compressor characteristic for compressor and close coupled valve is defined as

$$
\Psi_{e}\left(m, U_{1}\right)=\Psi_{c}\left(m, U_{1}\right)-\Psi_{v}(m)
$$

where $\Psi_{v}(m) p_{01}$ is the pressure drop across the CCV and

$$
\Psi_{c}\left(m, U_{1}\right)=\left(1+\frac{\eta_{i}\left(m, U_{1}\right) \Delta h_{0 c, \text { ideal }}}{T_{01} c_{p}}\right)^{\gamma / \gamma-1} .
$$

Assume $p_{0}, m_{0}$ to be the equilibrium values of pressure and mass flow as dictated by the intersection of the throttle and compressor characteristics, and $U_{d}$ to be the desired spool speed. Define the following error variables:

$$
\hat{p}=p-p_{0}, \quad \hat{m}=m-m_{0}, \quad \hat{U}=U_{1}-U_{d} .
$$

${ }^{3}$ It is worth noticing that a time varying $U$ is equivalent with a time varying $B$-parameter [10]. Greitzer's $B$-parameter as defined in [17] is given by $B=\left(U_{1} / 2 a_{01}\right) \sqrt{V_{p} / A_{1} L_{c}}$, where $V_{p}$ is the plenum volume and $L_{c}$ is the length of the compressor and duct. Using (43), a nonlinear differential equation for $B$ can be found. 
The equations of motion (45) are now transformed so that the origin becomes the equilibrium under study. Notice that no assumptions are made about the numeric values of $m_{0}$ and $p_{0}$, so that the equilibrium can be on either side of the surge line. By including the CCV (46), the model (45) can be written in the form

$$
\begin{aligned}
\dot{\hat{p}} & =\frac{a^{2}}{V_{p}}\left(\hat{m}-\hat{m}_{t}(\hat{p})\right) \\
\dot{\hat{m}} & =\frac{A}{L}\left(\left(\hat{\Psi}_{c}(\hat{m}, \hat{U})-\hat{\Psi}_{v}(\hat{m})\right) p_{01}-\hat{p}\right) \\
\dot{\hat{U}} & =\frac{D_{1}}{2 J}\left(\hat{\tau}_{t}-\hat{\tau}_{c}\right)
\end{aligned}
$$

where a hat denotes transformation to the new coordinates (48), and $(\hat{p} \hat{m} \hat{U})^{\mathrm{T}}=\left(\begin{array}{lll}0 & 0 & 0\end{array}\right)^{\mathrm{T}}$ is the equilibrium. From (10) it is known that

$$
\tau_{c}=\frac{D_{2}^{2} \sigma}{2 D_{1}}|m| U_{1}=\frac{D_{2}^{2} \sigma}{2 D_{1}} \operatorname{sgn}(m) m U_{1} .
$$

To avoid additional difficulties regarding continuity, a scaled hyperbolic tangent function is used in place of the signum function in the analysis

$$
\tau_{c}=\frac{D_{2}^{2} \sigma}{2 D_{1}} \tanh \left(\frac{m}{\varsigma}\right) m U_{1}
$$

where $\varsigma>0$ is a sufficiently large constant. The torque $\hat{\tau}_{c}$ is defined as

$$
\hat{\tau}_{c}=\tau_{c}-\tau_{c o}
$$

and calculated as

$$
\begin{aligned}
\tau_{c}= & \underbrace{\frac{D_{2}^{2} \sigma}{2 D_{1}} \tanh \left(\frac{m}{\varsigma}\right)\left(\hat{m}+m_{0}\right)\left(\hat{m} \hat{U}+\hat{m} U_{d}+m_{0} \hat{U}\right)}_{\hat{\tau}_{c}} \\
& +\underbrace{\frac{D_{2}^{2} \sigma}{2 D_{1}} \tanh \left(\frac{m}{\varsigma}\right) m_{0} U_{d}}_{\tau_{c 0}} .
\end{aligned}
$$

By choosing

$$
\hat{\tau}_{t}=\tau_{t}-\tau_{c o}
$$

the last equation in (49) follows from the last equation in (45).

Theorem 1: The surge control law

$$
\hat{\Psi}_{v}=k_{v} \hat{m}
$$

and the speed control law

$$
\begin{aligned}
\hat{\tau}_{t} & =-k_{p} \hat{U}-k_{i} \hat{I} \\
\dot{\hat{I}} & =\hat{U}
\end{aligned}
$$

where

$$
k_{p}>0, \quad k_{i}>0 \quad \text { and } \quad k_{v}>\sup _{\hat{U}, \hat{m}}\left\{\frac{\partial \hat{\Psi}_{c}(\hat{m}, \hat{U})}{\partial \hat{m}}\right\}+\delta_{1}
$$

and $\delta_{1}>0$, makes the origin of (49) semiglobal exponentially stable.
Proof: Define

$$
z \triangleq\left(\begin{array}{c}
\hat{U} \\
\hat{I}
\end{array}\right) \quad \text { and } \quad \boldsymbol{P} \triangleq\left(\begin{array}{cc}
\frac{2 J}{D_{1}} & \mu \\
\mu & k_{i}
\end{array}\right)
$$

where $\mu>0$ and $k_{i}>0$ are design parameters. Consider the following Lyapunov function candidate:

$$
V(\hat{p}, \hat{m}, \hat{U}, \hat{I})=\frac{1}{2}\left(V_{\hat{p}}+V_{\hat{m}}+V_{\text {spool }}\right)
$$

where

$$
V_{\hat{p}}=\frac{V_{p}}{a_{01}^{2} \rho_{01}} \hat{p}^{2}, \quad V_{\hat{m}}=\frac{L}{A_{1} \rho_{01}} \hat{m}^{2} \quad \text { and } \quad V_{\text {spool }}=\boldsymbol{z}^{\mathrm{T}} \boldsymbol{P} \boldsymbol{z} .
$$

It follows that $V$ is positive definite and radially unbounded, provided that $\mu$ is chosen such that $\boldsymbol{P}>0$, that is

$$
\mu<\sqrt{\frac{2 J k_{i}}{D_{1}}} .
$$

Calculating the time derivative of (59) along the solutions of (49) and accounting for (56) gives

$$
\begin{aligned}
\dot{V}= & \hat{m}\left(\hat{\Psi}_{c}(\hat{m}, \hat{U})-\hat{\Psi}_{v}(\hat{m})\right) \frac{p_{01}}{\rho_{01}}-\frac{1}{\rho_{01}} \hat{p} \hat{m}_{t}(\hat{p})-k_{p} \hat{U}^{2} \\
& +\mu \hat{U}^{2}-\frac{\mu k_{i} D_{1}}{2 J} \hat{I}^{2}-\frac{\mu k_{p} D_{1}}{2 J} \hat{U} \hat{I}-\hat{U} \hat{\tau}_{c}-\frac{\mu D_{1}}{2 J} \hat{I} \hat{\tau}_{c} .
\end{aligned}
$$

The last term in (62) can be upper bounded as

$$
\begin{aligned}
-\frac{\mu D_{1}}{2 J} \hat{I} \hat{\tau}_{c} & =-\frac{\mu D_{2}^{2} \sigma}{4 J}\left(\tanh \left(\frac{m}{\varsigma}\right) \hat{m} U+m_{0} \hat{U}\right) \hat{I} \\
& \leq \frac{\mu D_{2}^{2} \sigma}{4 J}\left(\frac{U_{m}}{2}\left(\frac{\hat{m}^{2}}{\eta_{1}}+\eta_{1} \hat{I}^{2}\right)+m_{0} \hat{U} \hat{I}\right)
\end{aligned}
$$

using (10), (44) and Young's inequality. The parameter $\eta_{1}>0$ can be chosen freely. The $\hat{U} \hat{\tau}_{c}$-term can be upper bounded as

$$
\begin{aligned}
-\hat{U} \hat{\tau}_{c}= & -\frac{D_{2}^{2} \sigma}{2 D_{1}} \tanh \left(\frac{m}{\varsigma}\right)\left(\left(\hat{m}+m_{0}\right) \hat{U}+\hat{m} U_{d}\right) \hat{U} \\
\leq & -\frac{D_{2}^{2} \sigma}{2 D_{1}} \tanh \left(\frac{m}{\varsigma}\right) m \hat{U}^{2} \\
& +\frac{D_{2}^{2} \sigma}{4 D_{1}}\left(\frac{\hat{m}^{2}}{\eta_{2}}+\eta_{2}\left(U_{d} \hat{U}\right)^{2}\right) .
\end{aligned}
$$

Now, (62) can be upper bounded as

$$
\begin{aligned}
\dot{V} \leq & \hat{m}\left(\hat{\Psi}_{c}(\hat{m}, \hat{U})-\hat{\Psi}_{v}(\hat{m})\right) \frac{p_{01}}{\rho_{01}} \\
& -\frac{D_{2}^{2} \sigma}{2 D_{1}} \tanh \left(\frac{m}{\varsigma}\right) m \hat{U}^{2} \\
& +\left(\frac{\sigma \mu D_{2}^{2} U_{m}}{8 J \eta_{1}}+\frac{D_{2}^{2} \sigma}{4 D_{1} \eta_{2}}\right) \hat{m}^{2} \\
& -\frac{1}{\rho_{01}} \hat{p} \hat{m}_{t}(\hat{p})-\boldsymbol{z}^{\mathrm{T}} \boldsymbol{R} \boldsymbol{z}
\end{aligned}
$$

where

$$
\boldsymbol{R}=\left(\begin{array}{cc}
k_{p}-\mu-\frac{D_{2}^{2} \sigma U_{d}^{2} \eta_{2}}{4 D_{1}} & \frac{\mu}{4 J}\left(D_{1} k_{p}-\frac{\sigma D_{2}^{2} m_{0}}{2}\right) \\
\frac{\mu}{4 J}\left(D_{1} k_{p}-\frac{\sigma D_{2}^{2} m_{0}}{2}\right) & \frac{\mu}{2 J}\left(k_{i} D_{1}-\frac{\sigma D_{2}^{2} U_{m} \eta_{1}}{4}\right)
\end{array}\right) .
$$



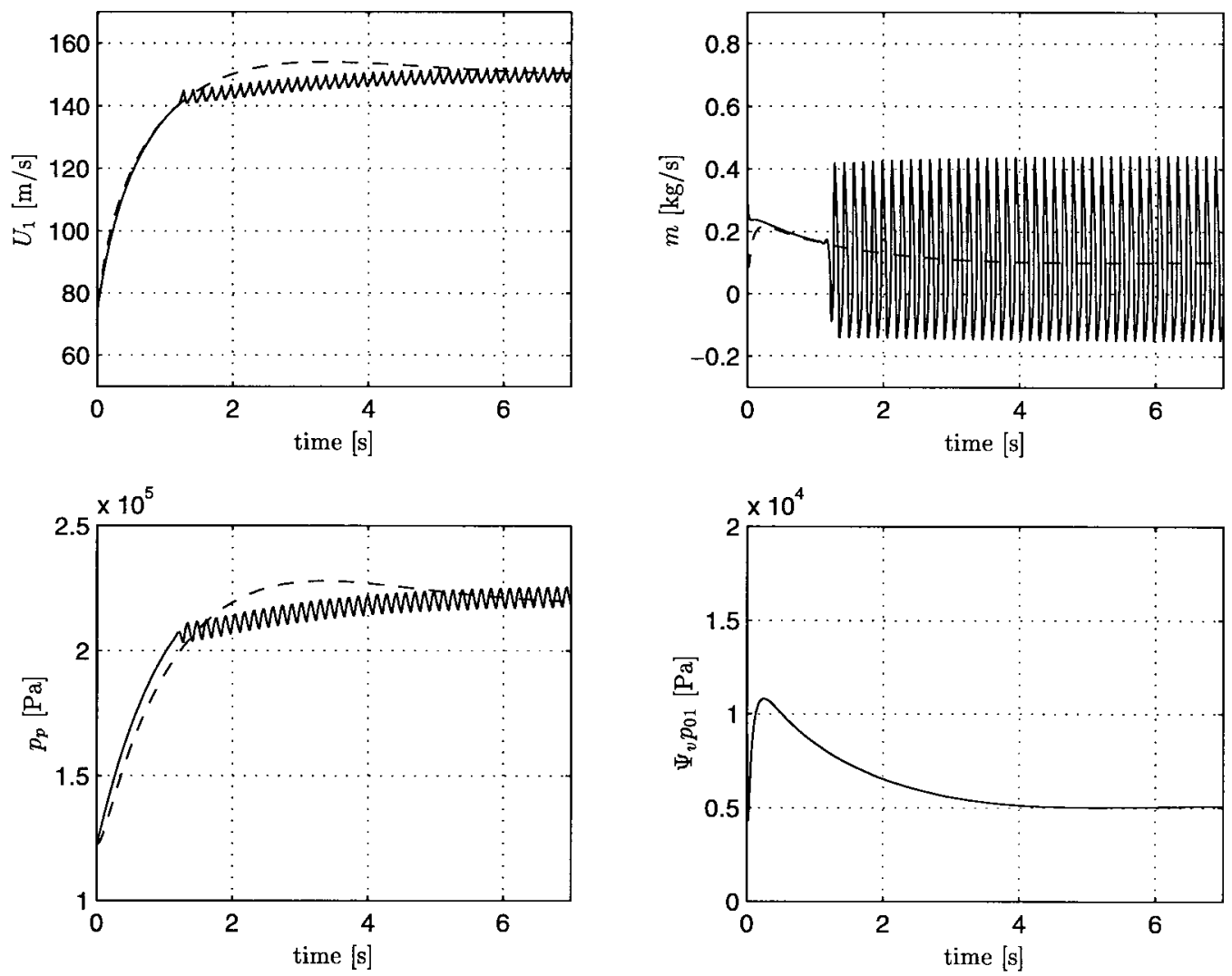

Fig. 10. Transient response of centrifugal compression system with annular diffuser. Without surge control, the compressor goes into surge. This is plotted with a solid line. The dashed lines are the system response when the CCV surge controller is in use.

Demanding $\boldsymbol{R}>0$ gives the following conditions on $k_{p}, \eta_{1}$, and $\mu$ :

$$
\begin{aligned}
k_{p} & >\frac{D_{2}^{2} \sigma U_{d}^{2} \eta_{2}}{4 D_{1}} \\
\eta_{1} & <\frac{4 k_{i} D_{1}}{\sigma D_{2} U_{m}}
\end{aligned}
$$

and

$$
\mu<\min \left\{\mu_{1}, \mu_{2}\right\}
$$

where

$$
\mu_{1}=k_{p}-\frac{D_{2}^{2} \sigma U_{d}^{2} \eta_{2}}{4 D_{1}}
$$

and

$$
\mu_{2}=\frac{\left(k_{p}-\frac{D_{2}^{2} \sigma U_{d}^{2} \eta_{2}}{4 D_{1}}\right)\left(k_{i} D_{1}-\frac{\sigma D_{2} U_{m} \eta_{1}}{4}\right)}{k_{i} D_{1}-\frac{\sigma D_{2} U_{m} \eta_{1}}{4}+\frac{1}{8 J}\left(D_{1} k_{p}-\frac{\sigma D_{2} m_{0}}{2}\right)^{2}} .
$$

It is assumed that $\hat{m}_{t}$ satisfies the sector condition

$$
\hat{p} \hat{m}_{t}(\hat{p})>\delta_{2} \hat{p}^{2}
$$

that is, the throttle is assumed passive as in [26]. As $\hat{p} \hat{m}_{t}(\hat{p})$ is of order $\frac{3}{2}$ in $\hat{p},(72)$ does not hold globally. However for a given $\hat{p}_{\max }$ such that

$$
|\hat{p}(t)| \leq p_{m} \quad \forall t>0
$$

it will always be possible to chose $\delta_{2}$ small enough for (72) to hold for $|\hat{p}(t)| \leq \hat{p}_{\max }$. Now, the CCV pressure drop $\Psi_{v}(\hat{m})$ is to be chosen such that for the first term in (65), the condition

$$
-\hat{m}\left(\hat{\Psi}_{c}(\hat{m}, \hat{U})-\hat{\Psi}_{v}(\hat{m})\right) \frac{p_{01}}{\rho_{01}}>0 \quad \forall \hat{U}
$$

is satisfied. Since $\left(p_{01} / \rho_{01}\right)>0$, sufficient conditions for (74) to hold is

$$
\begin{gathered}
-\left.\left(\hat{\Psi}_{c}(\hat{m}, \hat{U})-\hat{\Psi}_{v}(\hat{m})\right)\right|_{\hat{m}=0}=0 \quad \text { and } \\
\frac{\partial}{\partial \hat{m}}\left(-\hat{\Psi}_{c}(\hat{m}, \hat{U})+\hat{\Psi}_{v}(\hat{m})\right)>0 .
\end{gathered}
$$

It can be recognized that

$$
\begin{aligned}
\hat{\Psi}_{c}(0, \hat{U}) & =\Psi_{c}\left(m_{0}, U_{d}\right)-\Psi_{c}\left(m_{0}, U_{d}\right)=0 \\
\hat{\Psi}_{v}(\hat{m}) & =k_{v} \hat{m} \Rightarrow \hat{\Psi}_{v}(0)=0
\end{aligned}
$$

and thus (75) is satisfied. From (76), we get

$$
-\frac{\partial}{\partial \hat{m}} \hat{\Psi}_{c}(\hat{m}, \hat{U})+k_{v}>0
$$

and it follows that choosing $k_{v}$ according to

$$
k_{v}>\sup _{\hat{U}, \hat{m}}\left\{\frac{\partial \hat{\Psi}_{c}(\hat{m}, \hat{U})}{\partial \hat{m}}\right\}
$$




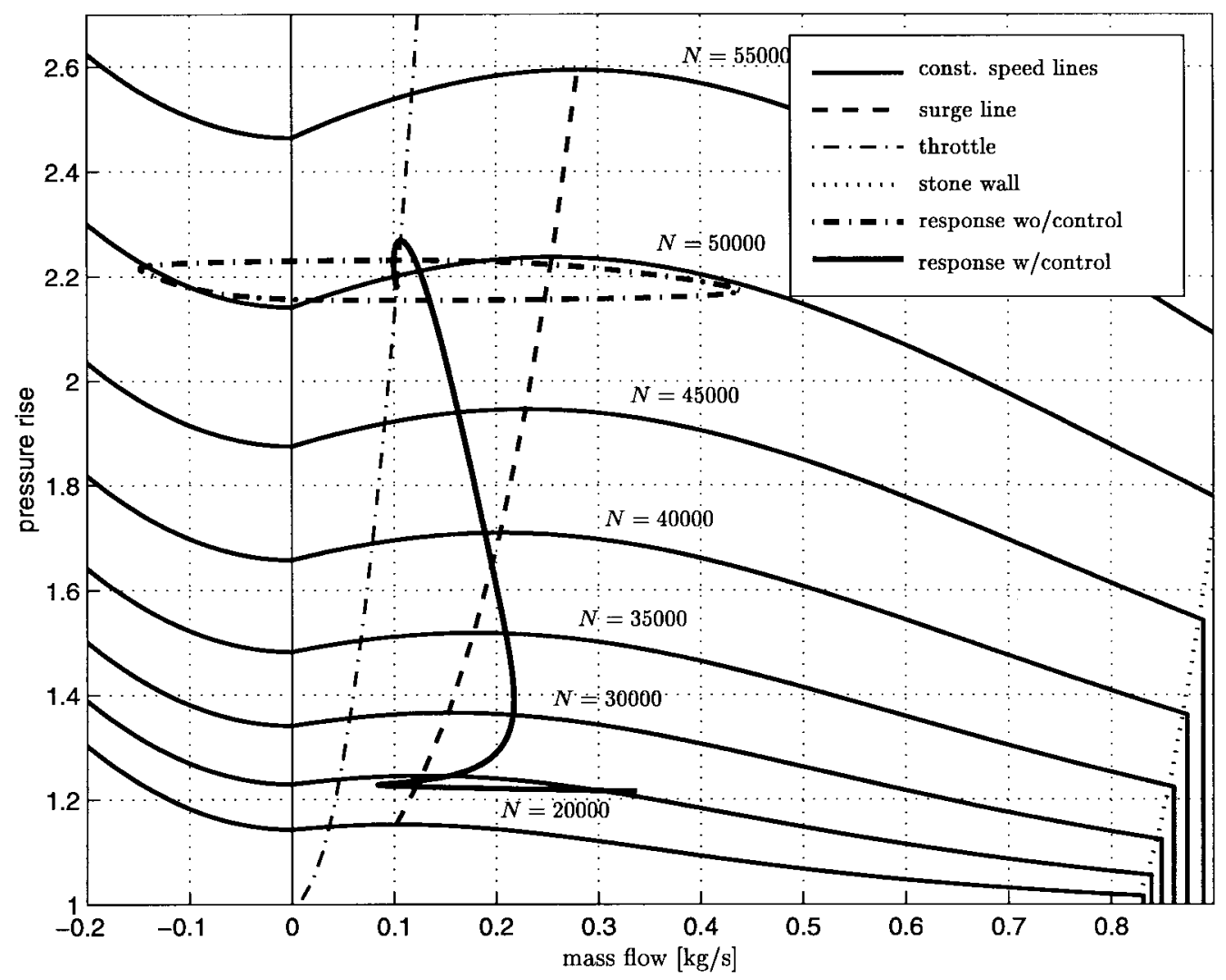

Fig. 11. ( $\left.m(t), p(t) / p_{01}\right)$-trajectories plotted together with the compressor characteristic. $N$ is the compressor speed in $\mathrm{r} / \mathrm{min}$.

guarantees that (76), and thereby (74) is satisfied. Moreover, if $k_{v}$ is chosen as

$$
k_{v}>\sup _{\hat{U}, \hat{m}}\left\{\frac{\partial \hat{\Psi}_{c}(\hat{m}, \hat{U})}{\partial \hat{m}}\right\}+\delta_{1}
$$

where $\delta_{1}>0$, we get $\hat{m} \Psi_{v}(\hat{m})>\delta_{1} \hat{m}^{2}$, and (74) is modified to

$$
-\hat{m}\left(\hat{\Psi}_{c}(\hat{m}, \hat{U})-\hat{\Psi}_{v}(\hat{m})\right) \frac{p_{01}}{\rho_{01}}>\frac{p_{01}}{\rho_{01}} \delta_{1} \hat{m}^{2} \quad \forall \hat{U}
$$

Consequently $\dot{V}$ can now be upper bounded as

$$
\begin{aligned}
\dot{V} \leq & -\left(\frac{p_{01}}{\rho_{01}} \delta_{1}-\frac{\sigma \mu D_{2}^{2} U_{m}}{8 J_{\eta_{1}}}-\frac{D_{2}^{2} \sigma}{4 D_{1} \eta_{2}}\right) \hat{m}^{2} \\
& -\delta_{2} \hat{p}^{2}-\frac{1}{2} \boldsymbol{z}^{\mathrm{T}} \boldsymbol{R} \boldsymbol{z} \quad \forall ; \hat{m}, \hat{p}, \boldsymbol{z} .
\end{aligned}
$$

We now set out to compare the coefficients of $V$ and $\dot{V}$. The cross terms in $\hat{U}$ and $\hat{I}$ are upper bounded using Young's inequality

$$
\begin{aligned}
\mu \hat{U} \hat{I} & \leq \frac{\mu}{2}\left(\frac{\hat{U}^{2}}{\eta_{3}}+\eta_{3} \hat{I}^{2}\right) \\
-\frac{\mu\left(4 D_{1} k_{p}-\sigma D_{2}^{2} m_{0}\right)}{2 J} \hat{U} \hat{I} \leq & \frac{\mu\left(4 D_{1} k_{p}-\sigma D_{2}^{2} m_{0}\right)}{4 J} \\
& \cdot\left(\frac{\hat{U}^{2}}{\eta_{4}}+\eta_{4} \hat{I}^{2}\right)
\end{aligned}
$$

where $\eta_{3}>0$ and $\eta_{4}>0$ are constants that can be chosen freely. Using (84) and (85) and comparing the coefficients in (59) and (83), it can be recognized that if the following inequalities are satisfied for some $\alpha>0$ :

$$
\begin{aligned}
\delta_{1}-\frac{\sigma \mu D_{2}^{2} U_{m} \rho_{01}}{8 J \eta_{1} p_{01}}-\frac{D_{2}^{2} \sigma \rho_{01}}{4 D_{1} \eta_{2} p_{01}}>\alpha \frac{L}{A \rho_{01}} \\
\delta_{2}>\alpha \frac{V_{p}}{a_{01}^{2} \rho_{01}} \\
k_{p}-\mu-\frac{\mu\left(4 D_{1} k_{p}-\sigma D_{2}^{2} m_{0}\right)}{4 J \eta_{4}}>\alpha\left(\frac{J}{D_{1}}+\frac{\mu}{2 \eta_{3}}\right) \\
\mu k_{i}-\frac{\mu\left(4 D_{1} k_{p}-\sigma D_{2}^{2} m_{0}\right)}{4 J} \eta_{4}>\alpha\left(\frac{k_{i}}{D_{1}}+\frac{\alpha \eta_{3}}{2}\right)
\end{aligned}
$$

the following holds:

$$
\dot{V} \leq-\alpha V \Rightarrow V(t) \leq V(0) e^{-\alpha t} .
$$

If $\eta_{1}$ is chosen according to (68), and $\delta_{1}$ is chosen according to

$$
\delta_{1}>\frac{\sigma \mu D_{2}^{2} U_{m} \rho_{01}}{8 J \eta_{1} p_{01}}+\frac{D_{2}^{2} \sigma \rho_{01}}{4 D_{1} \eta_{2} p_{01}}
$$

and $k_{v}$ is chosen so that $k_{v}>\sup _{\hat{U}, \hat{m}}\left\{\partial \hat{\Psi}_{c}(\hat{m}, \hat{U}) / \partial \hat{m}\right\}+$ $\delta_{1}$, and $\mu$ is chosen according to (61) and (69), then the inequalities (86)-(89) are satisfied for some $\alpha>0$. By (90) the origin of (49) is exponentially stable. Due to assumption (73), the stability result holds whenever $|\hat{p}(0)| \leq p_{m}$, and thus the origin is semiglobal exponential stable.

Notice that the parameter $\alpha$ can be used to calculate a lower bound on the convergence rate of the system. 

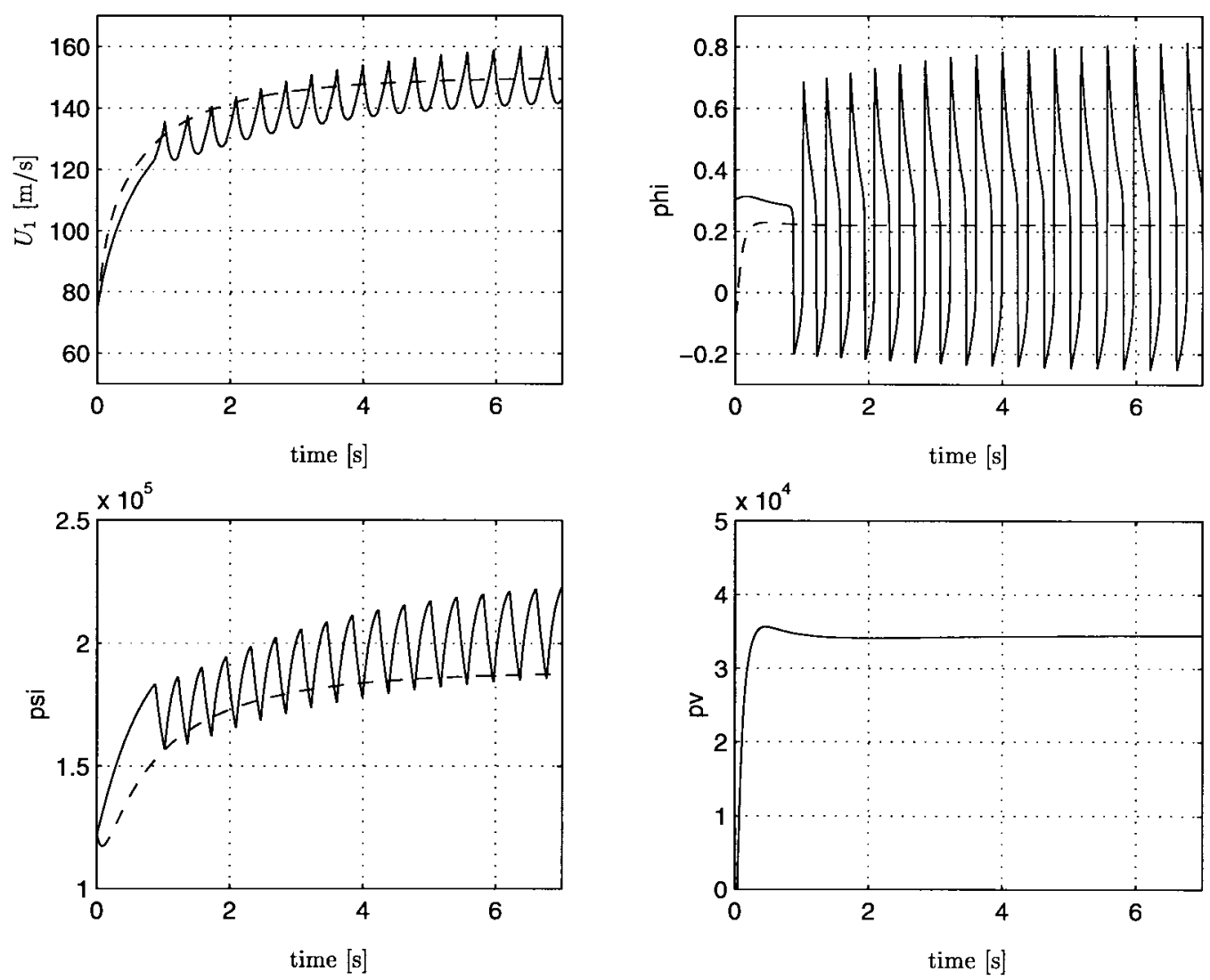

Fig. 12. Transient response of centrifugal compression system with vaned diffuser. Without surge control, the compressor goes into surge. This is plotted with a solid line. The dashed lines are the system response when the CCV surge controller is in use.

\section{SIMULATIONS}

The two cases of annular and vaned diffuser are now simulated with and without surge control.

\section{A. Annular Diffuser}

In Fig. 10 the response (solid lines) of the compression system during surge is shown. The set point for compressor speed was $U_{1 d}=150 \mathrm{~m} / \mathrm{s} \Rightarrow N \approx 50.000 \mathrm{r} / \mathrm{min}$ and the speed control law (56) parameters were set to $k_{p}=0.1$ and $k_{i}=0.07$. The throttle gain was set to $k_{t}=0.0003$ which gives an unstable equilibrium to the left of the surge line. Notice the oscillations in speed $U_{1}$. These variations in spool speed was first described in [8]. The limit cycle arising from this simulation is shown in Fig. 11, where the pressure rise $p_{p} / p_{01}$ has been plotted versus the mass flow together with the compressor characteristic, the throttle line and the surge line. As can be seen, the compressor undergoes deep surge oscillations.

Now, the surge controller (55) is used with $k_{v}=0.5$. The speed set point, speed controller parameters and throttle gain are as before. The results are shown in Fig. 10 (dashed lines). The desired speed is reached and the surge oscillations are eliminated. As previously mentioned there is a loss associated with the CCV control approach. The pressure drop over the valve is shown in the lower right corner of Fig. 10. At equilibrium the pressure drop for this particular case is at $5 \mathrm{kPa}$. Compared to the pressure rise over the compressor at this equilibrium, well over $200 \mathrm{kPa}$ this seems little when taken into account that the compressor now is operating in an area of the compressor map previously not possible. The CCV loss is dependent on the controller gain $k_{v}$. In this simulation the gain was set to $k_{v}=0.5$ to dominate the maximum positive slope of the compressor characteristic. The resulting trajectory of this simulation in also plotted in Fig. 11. The equilibrium is located somewhat below the intersection of the throttle line and the $N=50000$ line of the compressor characteristic. This is due to the pressure drop over the CCV.

\section{B. Vaned Diffuser}

The response of the compression system with speed control only is shown in Fig. 12 (solid lines). The speed control parameters were set to $k_{p}=0.1$ and $k_{i}=0.07$, and the throttle gain was set at $\gamma=0.00075$. As the vaned diffuser gives a steeper and more narrow characteristic, the amplitude of the pressure oscillations is larger than for the annular diffuser. This is also the case for the speed and mass flow oscillations.

When the surge control is in use, we get the response plotted with dashed lines in Fig. 12, and as can be seen the oscillations are avoided at the cost of a pressure loss over the CCV. Since the positive slope of the compressor characteristic is larger in this case compared to the annular diffuser, this pressure drop is also larger. However, a pressure drop of $35 \mathrm{kPa}$ over the valve is still less than the pressure rise of $180 \mathrm{kPa}$ over the compressor. 
By comparing the in-surge response of the two cases, it is seen that the frequency of the surge oscillations is lower for the vaned diffuser $(3 \mathrm{~Hz})$, than for the annular diffuser $(7 \mathrm{~Hz})$. This is in accordance with [18] and [33] where it is shown that the surge frequency depends on the slope of the compressor characteristic in such a way that a steeper slope leads to lower frequency, and a less steep slope leads to higher frequency.

\section{CONCLUSION}

A surge control law and a PI speed control law for a centrifugal compression system have been developed. The modeling of the compressor characteristic was based on energy losses in the compressor stage. Incidence and friction losses in the impeller and the diffuser were considered in addition to other losses. A close coupled valve was chosen as an actuator for the surge control. Using Lyapunovs method, the systems equilibrium was shown to be semiglobal exponentially stable. Through simulations it was confirmed that the compressor can operate stable and reach desired speed in the previous unstable area to the right of the surge line in the compressor map.

From a surge control point of view, the main difference between the annular and vaned diffusers are the steeper slope of the compressor characteristic. A consequence of this is that if a CCV is used to control surge, a greater pressure drop must be accepted over the valve in the case of a vaned diffuser than in the case of an annular diffuser.

\section{REFERENCES}

[1] O. E. Baljé, "A contribution to the problem of designing radial turbomachines," Trans. ASME, vol. 74, pp. 451-472, 1952.

[2] H. Cohen, G. F. C. Rogers, and H. I. H Saravanamuttoo, Gas Turbine Theory, 4th ed. Essex, U.K.: Longman, 1996.

[3] N. A. Cumpsty, Compressor Aerodynamics. Essex, U.K.: Longman, 1989.

[4] I. J. Day, "Axial compressor performance during surge," J. Propulsion Power, vol. 10, no. 3, pp. 329-336, 1994.

[5] S. L. Dixon, Thermodynamics of Turbomachinery, 3rd ed. Oxford, U.K.: Pergamon, 1978.

[6] J. L. Dussourd, G. W. Pfannebecker, and S. K. Singhania, "An experimental investigation of the control of surge in radial compressors using close coupled resistances," J. Fluids Eng., vol. 99, pp. 64-76, 1977.

[7] H. W. Emmons, C. E. Pearson, and H. P. Grant, "Compressor surge and stall propagation," Trans. ASME, vol. 77, pp. 455-469, 1955.

[8] K. M. Eveker and C. N. Nett, "Model development for active surge control rotating stall avoidance in aircraft gas turbine engines," in Proc. 1991 Amer. Contr. Conf., 1991, pp. 3166-3172.

[9] T. B. Ferguson, The Centrifugal Compressor Stage. London, U.K.: Butterworths, 1963.

[10] D. A. Fink, N. A. Cumpsty, and E. M. Greitzer, "Surge dynamics in a free-spool centrifugal compressor system," J. Turbomachinery, vol. 114, pp. 321-332, 1992.

[11] A. M. Foss, R. P. G. Heath, P. Heyworth, J. A. Cook, and J. McLean, "Thermodynamic simulation of a turbo-charged spark ignition engine for electric control development," in Proc. 7th IMechE Int. Conf. Automotive Electron., London, U.K., Oct. 1989, Paper C391/044, pp. 195-202.

[12] J. T. Gravdahl and O. Egeland, "A Moore-Greitzer axial compressor model with spool dynamics," in Proc. 36th IEEE Conf. Decision Contr. San Diego, CA, Dec. 1997, pp. 4714-4719.

[13] _ "Compressor surge control using a close-coupled valve and backstepping," in Proc. 1997 Amer. Contr. Conf., Albuquerque, NM, June 1997.

[14] _ "Passivity based compressor surge control using a close-coupled valve," in Proc. 1997 COSY Wkshp. Contr. Nonlinear Uncertain Syst., A. Isidori and F. Allgöwer, Eds., Zurich, Switzerland, Jan. 1997, pp. 139-143.

[15] , "Speed and surge control for a low-order centrifugal compressor model," in Proc. 1997 Int. Conf. Contr. Applicat., Hartford, CT, Oct. 1997, pp. 344-349.
[16] _ Compressor Surge and Rotating Stall: Modeling and Control, Advances in Industrial Control. London, U.K.: Springer-Verlag, 1999.

[17] E. M. Greitzer, "Surge and rotating stall in axial flow compressors, Part I: Theoretical compression system model," J. Eng. Power, vol. 98, pp. 190-198, 1976.

[18] _ _ "The stability of pumping systems-The 1980 Freeman scholar lecture," J. Fluids Eng., vol. 103, pp. 193-242, 1981

[19] K. E. Hansen, P. Jørgensen, and P. S. Larsen, "Experimental and theoretical study of surge in a small centrifugal compressor," J. Fluids Eng., vol. 103, pp. 391-394, 1981.

[20] J. P. Johnston and R. C. Dean, "Losses in vaneless diffusers of centrifugal compressors and pumps. Analysis, experiment and design," J. Eng. Power, Jan. 1966, pp. 49-62

[21] W. M. Jungowski, M. H. Weiss, and G. R. Price, "Pressure oscillation occurring in a centrifugal compressor system with and without passive and active surge control," J. Turbomachinery, vol. 118, pp. 29-40, 1996.

[22] J. A. Lorett and S. Gopalakrishnan, "Interaction between impeller and volute of pumps at off-design conditions," J. Fluids Eng., vol. 108, pp. 12-18, Mar. 1986

[23] A. E. Nisenfeld, Centrifugal Compressors: Principles of Operation and Control. Instrument Soc. Amer., 1982.

[24] R. C. Pampreen, "Small turbomachinery compressor and fan aerodynamics," J. Eng. Power, vol. 95, pp. 251-256, July 1973

[25] J. E. Pinsley, G. R. Guenette, A. H. Epstein, and E. M. Greitzer, "Active stabilization of centrifugal compressor surge," J. Turbomachinery, vol. 113, pp. 723-732, 1991.

[26] J. S. Simon and L. Valavani, "A Lyapunov based nonlinear control scheme for stabilizing a basic compression system using a close-coupled control valve," in Proc. 1991 Amer. Contr. Conf., 1991, pp. 2398-2406.

[27] J. S. Simon, L. Valavani, A. H. Epstein, and E. M. Greitzer, "Evaluation of approaches to active compressor surge stabilization," J. Turbomachinery, vol. 115, pp. 57-67, 1993.

[28] H.-H. Wang and M. Krstić, "Control of deep hysteresis compressors under limited actuator bandwidth," in Proc. 1997 Int. Conf. Contr. Applicat., Hartford, CT, Oct. 1997, pp. 657-662

[29] N. Watson and M. S. Janota, Turbocharging the Internal Combustion Engine. New York: MacMillan, 1982.

[30] F. M. White, Fluid Mechanics, 2nd ed. New York: McGraw-Hill, 1986

[31] A. Whitfield and F. J. Wallace, "Study of incidence loss models in radial and mixed-flow turbomachinery," in Proc. Conf. Heat Fluid Flow in Steam and Gas Turbine Plant, Univ. Warwick, Coventry, U.K., Apr. 1973, pp. 122-12.

[32] A. Whitfield and F. J. Wallace, "Performance prediction for automotive turbocharger compressors," Proc. Inst. Mech. Eng., vol. 189, no. 12, pp. 59-67, 1975

[33] F. Willems, "Modeling and control of compressor flow instabilities," Eindhoven Univ. Technol., The Netherlands, Tech. Rep. WFW 96.151, 1996.

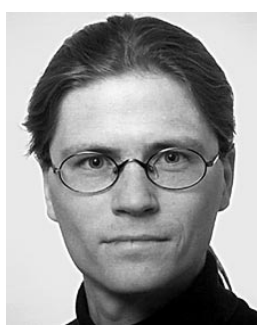

Jan Tommy Gravdahl (S'94-M'98) born in Norway in 1969. He received the Siv.Ing. degree in electrical engineering in 1994 and the Dr.Ing. degree in engineering cybernetics in 1998, both from The Norwegian University of Science and Technology (NTNU), Trondheim. His doctoral dissertation was titled "Modeling and control of surge and rotating stall in compressors."

$\mathrm{He}$ is currently a Postdoctoral Fellow at the Department of Engineering Cybernetics, NTNU. His research interests include nonlinear control with applications to mechanical systems, topics related to compressor modeling and control.

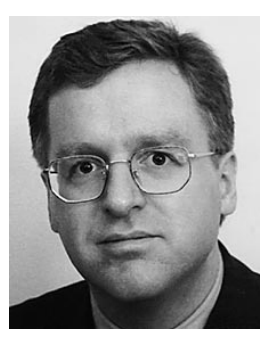

Olav Egeland (S'85-M'86) was born in Trondheim, Norway, in 1959. He received the Siv.Ing. degree in 1984 and the Dr.Ing. degree in 1987 in electrical engineering from the Norwegian University of Science and Technology, Trondheim.

He became Assistant Professor in 1987 and Professor in 1989 at the Department of Engineering Cybernetics, Norwegian University of Science and Technology, where he is currently Head of Department. The academic year 1988 to 1989 he was a Visiting Scientist at the German Aerospace Research Establishment (DLR) in Oberpfaffenhofen. His research interests include the modeling and control of nonlinear mechanical systems with focus on industrial applications.

Dr. Egeland is Associate Editor of IEEE Transactions on Automatic Control and received the Automatica Prize Paper Award in 1996. 